\title{
Implementación Computacional de la Transformada Fraccional de Fourier Discreta
}

\author{
Herbert E. Rojas ${ }^{(1) *}$, Camilo A. Cortés ${ }^{(2)}$, Diego F. Ramírez ${ }^{(3)}$ \\ (1) Universidad Distrital Francisco José de Caldas, Proyecto Curricular en Ingeniería Eléctrica, Grupo de \\ Compatibilidad e Interferencia Electromagnética - GCEM, Cra 7 № $40-53$, piso 5, Bogotá D.C.-Colombia. \\ (e-mail: herojasc@udistrital.edu.co) \\ (2) Universidad Nacional de Colombia, Departamento de Ingeniería Eléctrica y Electrónica, Grupo de \\ Investigación en Compatibilidad Electromagnética - EMC-UN, Laboratorio de Compatibilidad \\ Electromagnética, Edif. 411, Ciudad Universitaria, Bogotá D.C.-Colombia \\ (e-mail: caacortesgu@unal.edu.co) \\ (3) Universidad Nacional de Colombia, Laboratorio de Compatibilidad Electromagnética, Edif. 411, \\ Ciudad Universitaria, Bogotá D.C.-Colombia (e-mail: dframirezj@unal.edu.co)
}

* Autor a quien debe ser dirigida la correspondencia

Recibido May. 15, 2014; Aceptado Jul. 22, 2014; Versión final recibida Ago. 4, 2014

\begin{abstract}
Resumen
Este artículo presenta una introducción a la transformada fraccional de Fourier (FRFT) y describe el proceso para implementar la transformada fraccional de Fourier Discreta (DFRFT) basada en la descomposición espectral del operador matricial de la transformada discreta de Fourier (DFT). En la última década, la FRFT ha sido considerada como una herramienta útil para el procesamiento y tratamiento de señales. En el desarrollo teórico de la FRFT, se han presentado varias definiciones de su versión discreta (DFRFT). Sin embargo, pocas definiciones de la DFRFT entregan resultados similares a los que presenta la FRFT sin perder sus propiedades más importantes. Por lo tanto, en este trabajo se hacen algunas aclaraciones teóricas con respecto a trabajos previos, y se muestra la relación existente entre la FRFT y la DFRFT. Finalmente, con el fin de promover el uso de esta transformación dentro de la comunidad de procesamiento de señales, se describen algunos algoritmos de la DFRFT con bajos costos computacionales.
\end{abstract}

Palabras clave: transformada fraccional de Fourier, transformada discreta de Fourier, expansión espectral, vectores y valores propios

\section{Computational Implementation of the Discrete Fractional Fourier Transform}

\begin{abstract}
This paper provides an introduction of the fractional Fourier transform (FRFT) and describes the process of implementing the discrete fractional Fourier transform (DFRFT) based on the spectral decomposition of the discrete Fourier transform (DFT) matrix operator. In the last decade, the FRFT has been considered as a useful tool for signal processing applications. In the theoretical evolution of FRFT, many definitions of the DFTFT have been proposed in the literature. However, few definitions of the DFRFT present similar results to those provided by the FRFT without losing its most important properties. Therefore, some theoretical clarifications are made with respect to previous works. Moreover, the relationship between the FRFT and the DFRFT is also shown. Finally, in order to promote the use of this transform in the signal processing community, some algorithms of the DFRFT with low computational costs are described.
\end{abstract}

Keywords: fractional Fourier transform, discrete Fourier transform, spectral expansion, eigenvectors and eigenvalues 


\section{INTRODUCCIÓN}

La transformación fraccional de Fourier continua (FRFT) es una representación tiempo-frecuencia (TFR) y es una generalización de la transformada clásica de Fourier (FT). Introducida por Namias (1980) y estructurada matemáticamente por McBride y Kerr (1987), la FRFT y su potencial permanecieron ocultas a los ojos de la comunidad científica en procesamiento de señales hasta la década de 1990. A partir de entonces, la FRFT ha generado gran interés por su amplia variedad de aplicaciones en áreas tales como: óptica, imágenes, señales variantes en el tiempo, diseño de filtros, encriptación de información, detección de singularidades, radar, sonar y telecomunicaciones, entre otros (Sejdić et al., 2011).

Con el avance del procesamiento digital de señales, el uso de mejores equipos para el muestreo y digitalización de información y el constante desarrollo de métodos eficientes de cómputo, la aplicación de la FRFT en diferentes campos de la ingeniería ha ganado gran importancia en las últimas dos décadas, lo cual ha provocado un incremento acelerado en el estudio de la FRFT y su discretización. En la actualidad se busca que la versión discreta de la FRFT (DFRFT) cumpla con los siguientes requisitos: (a) ser un operador unitario, (b) tener orden aditivo, (c) ser una generalización de la transformada discreta de Fourier (DFT), y (d) aproximar de manera adecuada a la FRFT.

En la actualidad existen diversas definiciones de la DFRFT, las cuales generalmente se obtienen a partir del uso de una matriz discreta que representa el operador FRFT. Esta matriz puede ser obtenida por diferentes métodos que agrupan varios tipos de DFRFT. Entre los métodos más difundidos se tienen: la DFRFT por muestreo de la FRFT, la DFRFT por suma ponderada o combinación lineal y la DFRFT por descomposición de vectores y valores propios (Tao et al., 2006; Sejdić et al., 2011). Adicionalmente, al revisar la literatura se encuentra que en Ozaktas et al. (1996) no se cumple con los dos primeros requisitos que debe cumplir la DFRFT, en Bultheel y Sulbaran (2004) se presentan varios algoritmos que tienen problemas de eficiencia computacional y pérdida de información. Recientemente, Pei et al. (2006) presenta un método basado en la estimación de matrices tridiagonales que computan con la DFT para obtener los vectores propios de la matriz DFRFT, Koc et al. (2008) propone una definición de la DFRFT basada en la descomposición de la transformación lineal canónica (LCT). Esta definición aunque robusta y generalizada, no ajusta adecuadamente para algunos órdenes fraccionales. Finalmente, Pei y Hsue (2009) presentan un algoritmo de la DFRFT que usa una matriz operador (núcleo) con vectores y valores propios de la DFT de naturaleza aleatoria

Aunque estas definiciones de la transformación fraccional discreta de Fourier, DFRFT, pueden ser usadas con mayor o menor confianza según el tipo de aplicación, muy pocas referencias muestran un proceso detallado para el desarrollo e implementación de sus algoritmos. Este artículo presenta una introducción a la transformación fraccional de Fourier (FRFT) y describe el proceso para implementar la transformación fraccional de Fourier Discreta (DFRFT) basada en la expansión espectral del operador matricial de la transformación discreta de Fourier (DFT) y el cálculo de sus valores y vectores propios, los cuales han sido estudiados previamente por Dickinson y Steiglitz (1982), McClellan y Parks (1972) y Pei et al., (1999). Estos vectores propios pueden ser calculados usando una matriz de conmutación o a partir de la discretización de las funciones normalizadas de Hermite-Gauss (HG-F), las cuales son conocidas por ser funciones propias de la FRFT. Adicionalmente, se muestra que esta definición de la DFRFT es consistente para diversos tipos de señales y cumple con los requerimientos antes mencionados y cuyos resultados guardan relación con la FRFT continua.

El propósito de este artículo, además de describir el proceso para desarrollar algoritmos confiables y eficientes, es promover el uso y aplicación de la DFRFT en áreas de ingeniería donde el procesamiento de señales hace parte importante del análisis de eventos o singularidades, como por ejemplo en los problemas abordados por Alarcon et al. (2005), Martinez et al. (2010) y Santamaria et al. (2012). La difusión y promoción de esta herramienta de procesamiento complementa el trabajo que ha sido desarrollado por los autores de este trabajo el cual se ha orientado hacia el análisis de perturbaciones electromagnéticas usando técnicas de procesamiento basadas en TFRs, como la transformación de ondeletas (wavelets) (Santamaria et al., 2012).

\section{BASES TEORICAS Y DEFINICIONES}

Con el propósito de establecer bases conceptuales que faciliten el proceso de implementación computacional de la transformada DFRFT es conveniente presentar en esta sección algunos conceptos y definiciones relacionados con la transformada fraccional de Fourier continua (FRFT), la transformación discreta de Fourier (DFT) y su representación matricial y analizar algunos teoremas necesarios para definir la DFRFT. 


\section{La transformación fraccional de Fourier continua (FRFT)}

La versión radial normalizada de la transformación de Fourier (FT) de una señal $x(t)$ se define como (Bracewell, 2000):

$\mathcal{F}[x(t)](\omega) \equiv X(\omega)=\frac{1}{\sqrt{2 \pi}} \int_{-\infty}^{\infty} x(t) e^{-j \omega t} d t$

Mientras la FT es interpretada como la rotación de una señal $x(t)$ en un ángulo $\pi / 2$ en el plano tiempofrecuencia (TFp), la FRFT puede ser interpretada como una rotación de la señal en cualquier ángulo $\alpha=$ $a \pi / 2$ en el mismo TFp (Almeida, 1994). Al ser una generalización de la FT, la FRFT de orden $a$ de una señal $x(t)$, denotada como $X_{a}(u)$, y su transformada inversa (IFRFT) pueden ser definidas a través de su representación integral de la siguiente manera (Almeida, 1994):

$\mathcal{F}^{a}[x(t)](u) \equiv X_{a}(u)=\int_{-\infty}^{\infty} x(t) B_{a}(t, u) d t \quad \mathcal{F}^{-a}\left[X_{a}(u)\right](t) \equiv x(t)=\int_{-\infty}^{\infty} X_{a}(u) B_{-a}(t, u) d u$

donde $0<|a|<2$ y $B_{a}(t, u)$ es el núcleo de integración de la FRFT, el cual es definido como (Almeida, 1994; Namias, 1980):

$B_{a}(t, u)=\left\{\begin{array}{cl}K_{\alpha} \exp \left[j\left(\frac{t^{2}+u^{2}}{2}\right) \cot \alpha-j u t \csc \alpha\right] & \text { para } a \neq 0 \pm 2 \pm 4 \ldots \\ \delta(t-u) & \text { para } a=0 \pm 4 \ldots \\ \delta(t+u) & \text { para } a= \pm 2 \pm 6 \ldots\end{array}\right.$

donde, $K_{\alpha}=\sqrt{(1-j \cot \alpha) / 2 \pi}$. De esta manera, cuando $a=1, B_{a}(t, u)=(\sqrt{1 / 2 \pi}) e^{-j t u}$ y la FRFT se reduce a la definición de la FT mostrada en (1). La definición dada en (2) es válida para cualquier valor real de $a$ y como se muestra en (3) puede ser extendida mediante la notación $\mathcal{F}^{a}[x(t)](u)=\mathcal{F}^{a} \bmod 4[x(t)](u)$.

En esta definición de la FRFT la variable independiente $(u)$, conocida como dominio fraccional de Fourier (FRFd), posee dos casos especiales: cuando $a=0, u$ se convierte en $t$ y cuando $a=\pi / 2, u$ se convierte en $\omega$. Las condiciones de existencia de la FRFT, así como sus propiedades y las de su núcleo de integración son expuestas en Namias, (1980), McBride y Kerr (1987) y Sejdić et al. (2011).

El núcleo de transformación (kernel) de la FRFT también puede ser expresado en términos de su expansión espectral como (Namias, 1980):

$B_{a}(t, u)=\sum_{n=0}^{\infty} \phi_{n}(u) e^{-j n a \pi / 2} \phi_{n}(t)$

donde, $\phi_{n}(t)$ son las funciones normalizadas de Hermite-Gauss (HG-F) de orden $n$, definidas como (Lebedev, 1972):

$\phi_{n}(t)=\left[1 /\left(2^{n} n ! \sqrt{\pi}\right)\right]^{1 / 2} H_{n}(t) \exp \left(-t^{2} / 2\right)=\mu_{n} H_{n}(t) \exp \left(-t^{2} / 2\right)$

donde, $n=\{0,1,2, \ldots n\} \in \mathbb{Z}$ es el orden de la función y $H_{n}(t)$ son los polinomios de Hermite. La equivalencia matemática entre (3) y (4) se muestra de manera detallada en Namias (1980).

Puesto que el núcleo de la FRFT depende de las HG-F, (2) puede ser reescrita de la siguiente manera:

$X_{a}(u)=\sum_{n=0}^{\infty} \phi_{n}(u)\left\{e^{-j n a \pi / 2} \int_{-\infty}^{\infty} x(t) \phi_{n}(t) d t\right\}$

Al existir en un dominio entre el tiempo y la frecuencia, la FRFT pertenece a un grupo de TFRs que han sido ampliamente usadas en el procesamiento de señales. Es por esto que la FRFT que posee relación con otras TFRs tales como: la distribución Wigner (WD), la transformación de ondeletas (WT), la transformación lineal canónica (CLT), la transformación local polinomial de Fourier (LPFT) y otras operaciones relacionadas con señales chirp (Ozaktas et al., 1994). 


\section{La transformación discreta de Fourier (DFT) y su representación matricial}

Debido a que en este artículo se utiliza la versión radial normalizada de la FT, es necesario dar una definición de la DFT que concuerde con (1). Siguiendo la metodología expuesta en (Hsu, 1995) se tiene que la DFT de una señal $x(t)$ se define como:

$\mathcal{F}(x[k])[m]=\frac{1}{\sqrt{N}} \sum_{k=-N / 2}^{N / 2-1} x[k] e^{-j(2 \pi k / N) m}, \quad$ donde $\quad x[k]=\{T s \sqrt{(N / 2 \pi)}\} x\left(k T_{S}\right)$

donde, $x[k]$ es la señal discretizada de $N$ muestras. Sí $T_{S}=\sqrt{2 \pi / N}, x[k]=x\left(k T_{S}\right)$ y coincidirá con la definición dada por el teorema del muestreo. Usando notación matricial, (7) puede ser expresada como:

$\mathbb{F}(x[k])[m]=X[m, k]=(1 / \sqrt{N})\left[\underline{x} \cdot \underline{w^{-k}}\right]$

donde, $\underline{x}$ es una matriz columna de $N$ muestras y $\underline{w^{-k}}$ es una matriz $N \times N$ de exponentes complejas. A partir de (8), se puede interpretar la DFT como un operador matricial $\underline{\mathbb{F}}$ cuya representación es (Hsu, 1995):

$\underline{\mathbb{F}}[m, k]=\left(\frac{1}{\sqrt{N}}\right) \sum_{k=0}^{N-1} \underline{w^{-k}}[m]=\left(\frac{1}{\sqrt{N}}\right) \sum_{k=0}^{N-1} e^{-j(2 \pi k / N) m}, \quad$ con $m, k=0,1,2, \ldots, N-1$

Por otra parte, la matriz $\underline{\mathbb{F}}$ puede expresarse aplicando el teorema de descomposición espectral, así (Roman, 2010):

$\underline{\mathbb{F}}[m, k]=\sum_{n=0}^{N-1} p_{n}[m]\left(\lambda_{n}\right) p_{n}[k], \quad$ con $m, k=0,1,2, \ldots, N-1$

donde, $p_{n}[k]$ es el $n$-esimo vector propio ortonormal de la representación matricial de la DFT dispuesto en columna y asociado al valor propio $\lambda_{n}$ correspondiente. De esta forma, el operador DFT es expresado de manera similar al núcleo $B_{a}$ de la FRFT mostrado en (4). Además de estas definiciones, la Tabla 1 muestra algunos conceptos adicionales que serán de utilidad en las siguientes secciones (Roman, 2010).

Tabla 1: Conceptos y teoremas necesarios para definir la DFRFT

- Definición 1: Un operador lineal $\mathcal{S}$ sobre $\mathbb{C}^{N}$, es unitario sí $\mathcal{S}^{*}=\mathcal{S}^{-1}$ y es normal sí $\mathcal{S}^{*} \mathcal{S}=\mathcal{S} \mathcal{S}^{*}$. Análogamente una matriz $\mathbb{A}$ se dice normal sí $\mathbb{A}^{*} \mathbb{A}=\mathbb{A} \mathbb{A}^{*}$.

- Teorema 1: Un operador $\mathcal{S}$ o una matriz $\mathbb{A}$ es diagonalizable, sí y solo sí existe una base ortonormal de $\mathbb{C}^{N}$ formada por vectores propios de $\mathcal{S} \circ \mathbb{A}$ en $\mathbb{C}^{N}$.

- Teorema 2: Si dos operadores $\mathcal{A}$ y $\mathcal{B}$ conmutan, es decir que $\mathcal{A B}=\mathcal{B} \mathcal{A}$, existirá un conjunto de vectores propios comunes entre $\mathcal{A}$ y $\mathcal{B}$.

Para la diagonalización de la matriz $\mathbb{F}$ es necesario estimar sus valores y vectores propios (Teorema 1). Puesto que la representación matricial de la DFT no posee representación única debido a que $\underline{\mathbb{F}}$ posee cuatro espacios vectoriales que corresponden a sus valores propios $\{1,-1, j,-j\}$ (McClellan y Parks, 1972), es necesario construir una matriz real y simétrica $\boldsymbol{U}$ que conmute con $\underline{\mathbb{F}}$. De esta manera, al estimar un conjunto de vectores propios de $U$, se pueden conocer los vectores propios de $\underline{\mathbb{F}}$ (Teorema 2). Esta matriz $\boldsymbol{U}$ es definida como (Dickinson y Steiglitz, 1982):

$U=\left[\begin{array}{ccccccc}2 & 1 & 0 & 0 & \ldots & 0 & 1 \\ 1 & 2 \cos \left(\frac{2 \pi}{N}\right) & 1 & 0 & \ldots & 0 & 0 \\ 0 & 1 & 2 \cos \left(\frac{4 \pi}{N}\right) & 1 & \ldots & 0 & 0 \\ \vdots & \vdots & \vdots & \vdots & \ddots & \vdots & \vdots \\ 1 & 0 & 0 & 0 & \ldots & 1 & 2 \cos \left(\frac{2 \pi(N-1)}{N}\right)\end{array}\right]$

La condición impuesta sobre la matriz $\boldsymbol{U}$ se debe a que este tipo de matrices posee valores propios $\lambda$ de multiplicidad 1, lo que garantiza que sus vectores propios sean diferentes y ortogonales (Roman, 2010). 


\section{VERSIONES DE LA DEFINICIÓN DE LA FRFT DISCRETA (DFRFT)}

De manera preliminar, la DFRFT puede ser definida como un operador $\mathbb{F}^{a}$ sobre un espacio vectorial de $n$-dimensiones, el cual debe satisfacer los siguientes requerimientos (C̄andan et al., 2000): (a) ser un operador unitario, (b) reducirse a la DTF cuando el orden a sea unitario, (c) tener orden aditivo de manera que $\underline{F}^{a} \underline{F}^{b}=\mathbb{F}^{a+b}$, y (d) aproximarse de manera adecuada a la FRFT. En la literatura se han clasificado diferentes versiones de la DFRFT (Tao et al., 2008). A continuación, se hace una breve revisión de cada una de ellas.

\section{DFRFT por muestreo}

Esta definición se basa en la discretización de las señales de entrada y de salida de la FRFT y la aplicación del concepto de interpolación de Nyquist (Bultheel, 2004; H. Ozaktas et al., 1996). Debido a que en su aplicación se pierden propiedades esenciales de la DFRFT como la reversibilidad y la aditividad, la DFRFT por muestreo normalmente no es considerada como una definición de la DFRFT sino como un algoritmo que discretiza la FRFT (Tao et al., 2008). Aunque no existe un método eficiente para demostrar que esta definición cumple con los cuatro requerimientos básicos que debe tener la DFRFT (Candan et al., 2000), se han propuesto algoritmos que permiten obtener resultados similares a los de la FRFT (Pei y Ding, 2000). Esto ha permitido usar esta definición de la DFRFT en aquellos casos en los que se quieren obtener resultados con alta precisión usando un algoritmo de bajos costos computacionales, pocas restricciones y que puede ser adaptado a un procesador digital de señales (DSP).

\section{DFRFT de suma ponderada o de combinación lineal}

En esta definición, la DFRFT de cualquier señal puede ser obtenida mediante el uso de DFRFT conocidas u órdenes fraccionales especiales. Esta definición calcula las potencias fraccionales de la representación matricial de la DFT como una combinación lineal de sus potencias enteras de la siguiente manera (Pei et al., 1999):

$\underline{\mathbb{F}}^{a}=\sum_{k=1}^{4} \theta_{k}(a) \underline{\mathbb{F}}^{k} \quad$ donde, $\quad \theta_{k}(a)=\frac{1}{4} \sum_{l=1}^{4} e^{j(\pi / 2)(a-k) l}$

Si $\underline{x}=x(n)$, es una señal discreta de $N$ muestras, la DFRFT de suma ponderada se define como:

$X_{a}=\theta_{1}(a) X[m]+\theta_{2}(a) x(-n)+\theta_{3}(a) X[-m]+\theta_{4}(a) x(n)$

donde, $X[m]$ es la DFT de la señal $x(n)$. Esta DFRFT cumple con el segundo requisito y puede evaluarse usando transformada rápida de Fourier (FFT). Sin embargo, no cumple con el cuarto requisito pues no aproxima de manera adecuada a la FRFT (Pei et al., 1999; Tao et al., 2008). Aun así, es el algoritmo con menor complejidad computacional y es usado para comprimir información y su reconstrucción se hace usando DFT inversa.

\section{DFRFT por descomposición espectral (descomposición de vectores propios)}

La DFRFT por descomposición espectral de la DFT ha sido definida por Candan et al. (2000) y Pei et al. (1999). Esta versión se basa en la descomposición de la representación matricial o núcleo de la DFT y utiliza conceptos relacionados con los valores propios de la matriz $\mathbb{F}$, sus multiplicidades y sus correspondientes sub-espacios vectoriales. Es importante resaltar que la descomposición espectral del núcleo de la DFT no es única, lo que representa una variedad de métodos para determinar sus valores y vectores propios (Dickinson y Steiglitz, 1982; McClellan y Parks, 1972). Es así como Cincotti et al. (1992) introduce un método para calcular los vectores propios de la DFT, pero no se obtienen los valores reales de estos vectores. Dickinson y Steiglitz (1982) y Candan et al. (2000) presentan un método para determinar un conjunto completo de vectores propios de la DFT a partir de la introducción de una matriz que conmuta con F (Teorema 2). Adicionalmente, Pei et al. (1999) muestra un método en el que se determinan un conjunto de vectores propios de la DFT con formas similares a las HG-F, las cuales como se mencionó en la sección anterior, son funciones propias del operador FRFT.

Debido a que esta definición de la DFRFT conserva ampliamente las propiedades de la FRFT y además, guarda relación directa con la DFT de la misma manera en que la FRFT lo hace con la FT (Tao et al., 2006), en las siguientes secciones de este trabajo se describe el proceso para implementar la DFRFT por descomposición espectral. Además, debido a los altos costos computacionales que posee el cálculo de esta definición de la DFRFT, en la sección 6 se proponen dos algoritmos adicionales que pueden ser usados para aplicaciones en tiempo real. 


\section{DEFINICION COMPACTA DE LA DFRFT POR DESCOMPOSICION ESPECTRAL}

Esta sección describe el proceso para obtener la DFRFT basada en la descomposición espectral. Este trabajo toma como punto de referencia las definiciones presentadas por Pei et al. (1999) y Candan et al. (2000). Sin embargo, se hace una exposición más detallada del proceso y se realizan algunos aportes que complementan y facilitan el desarrollo de un algoritmo confiable de la DFRFT.

\section{Cumplimiento de los requisitos para ser una definición válida de la DFRFT}

Sea $p_{k}[n]$ un vector propio ortonormal arbitrario dispuesto en columna y asociado al valor propio $\lambda_{k}$, ambos correspondientes a la matriz $\underline{\mathbb{F}}$ definida en (10), el operador DFRFT sobre $\mathbb{C}^{N}$ puede ser representado por una matriz $\underline{F}^{a}$, a través de una expansión análoga a la presentada en (4), de la siguiente manera:

$\underline{F}^{a}[m, n]=\sum_{k=0}^{N-1} p_{k}[m]\left(\lambda_{k}\right)^{a} p_{k}[n], \quad$ donde $n, m=1,2, \ldots, N$

Se observa que (14) cumple el primer requerimiento, pues los valores propios de la matriz $\mathbb{F}\left(\lambda_{k}=e^{j \pi k / 2}\right)$ son de magnitud unitaria (Dickinson y Steiglitz, 1982; McClellan y Parks, 1972). El segundo requerimiento también se cumple, pues cuando $a=1$ la ecuación (14) se reduce a la expansión espectral del operador DFT mostrado en (10).

Para demostrar que el tercer requerimiento se cumple es necesario definir una matriz $\boldsymbol{P}$ como una base ortonormal formada por los vectores propios de la matriz $\underline{\mathbb{F}}$ dispuestos en columna y la matriz diagonal $\boldsymbol{\Lambda}_{\boldsymbol{a}}$ formada por los valores propios de $\underline{\mathbb{F}}$. Con este arreglo, y teniendo en cuenta que $\boldsymbol{P}^{\mathbf{- 1}}=\boldsymbol{P}^{*}$, se puede definir que $P^{-1} \Lambda_{a} P=P^{*} \Lambda_{a} P$. Además:

$\underline{\mathbb{F}}^{a} \underline{\mathbb{F}}^{b}=\left(P^{*} \Lambda_{a} P\right)\left(P^{*} \Lambda_{b} P\right)=P^{*} \Lambda_{a} \Lambda_{b} P=P^{*} \Lambda_{a+b} P=\underline{\mathbb{F}}^{a+b}$

Esto muestra que la definición presentada en (14) cumple la propiedad de la aditividad. Respecto al cuarto requerimiento, se mostrará en la parte final de este trabajo (con ayuda de algunos ejemplos) que esta definición de la DFRFT guarda relación y aproxima de manera adecuada la FRFT.

Matriz de conmutación S a partir de las HG-F y la ecuación de Harper

Las HG-F, definidas en (5), son funciones propias del operador FT $(\mathcal{F})$, y también son la única solución de energía finita para la siguiente ecuación diferencial:

$\frac{d^{2} \gamma(t)}{d t^{2}}-t^{2} \gamma(t)=\lambda \gamma(t)$

donde, $\gamma(t)$ es una función dependiente del tiempo y $\lambda=-(2 n+1)$. Si $\Gamma(\omega)=\mathcal{F}[\gamma(t)](\omega)$ tiene convergencia uniforme, al derivar dos veces $\Gamma(\omega)$ respecto a $\omega$ se tiene que:

$\frac{d^{2} \Gamma(\omega)}{d \omega^{2}}=\frac{1}{\sqrt{2 \pi}} \int_{-\infty}^{\infty}\left[-t^{2} \gamma(t)\right] e^{-j \omega t} d t$

Aplicando FT inversa a ambos lados de (17) y usando el operador diferencial $\mathcal{D}_{x}^{n}=d^{n} / d x^{n}$ y el operador FT $\mathcal{F}$, esta ecuación se puede reescribir como:

$\mathcal{F}^{-1}\left\{\mathcal{D}_{\omega}^{2}(\mathcal{F}[\gamma(t)])\right\}=-t^{2} \gamma(t)$

Reemplazando (18) en (17) y utilizando adecuadamente el operador diferencial se tiene:

$\left[\mathcal{D}_{t}^{2}+\mathcal{F}^{-1} \mathcal{D}_{\omega}^{2} \mathcal{F}\right] \gamma(t)=\lambda \gamma(t)$

La ecuación (19) es ligeramente diferente de la ecuación $\left[\mathcal{D}^{2}+\mathcal{F D}^{2} \mathcal{F}^{-1}\right] \gamma(t)=\lambda \gamma(t)$ presentada por Candan et al. (2000) ya que en esta última no se distinguen los dominios del tiempo y la frecuencia en los operadores diferenciales. Si bien desde el punto de vista matemático esto es irrelevante, porque el dominio de la señal y el de su FT es el conjunto de los reales, desde el punto de vista físico estos dominios tienen una interpretación diferente lo que hace necesaria la distinción de las variables de cada operador diferencial. 
Puesto que las soluciones de una ecuación diferencial pueden ser estimadas mediante su discretización y el uso de la ecuación de Harper (HE) (Barker, 2000). (19) puede ser expresada para cada valor de $n=$ $\{0,1,2, \ldots, N-1\}$ como una suma discreta de Fourier a través de la relación matricial:

$\left(S-\omega_{0} \lambda I\right) C=0 \quad$ donde, $\omega_{0}=2 \pi / N$ y

$S=\left[\begin{array}{ccccccc}-2 & 1 & 0 & 0 & \cdots & 0 & 1 \\ 1 & 2 \cos \left(\frac{2 \pi}{N}\right)-4 & 1 & 0 & \cdots & 0 & 0 \\ 0 & 1 & 2 \cos \left(\frac{4 \pi}{N}\right)-4 & 1 & \cdots & 0 & 0 \\ \vdots & \vdots & \vdots & \vdots & \ddots & \vdots & \vdots \\ 1 & 0 & 0 & 0 & \cdots & 1 & 2 \cos \left(\frac{2 \pi(N-1)}{N}\right)-4\end{array}\right]$

$\& \quad C=\left[\begin{array}{c}C_{0} \\ C_{1} \\ C_{2} \\ \cdots \\ C_{N-1}\end{array}\right]$

El proceso que permite estimar este sistema matricial está disponible como material complementario, tal y como se explica al final de este artículo.

Si se compara la matriz $S$ en (20) con la matriz $U$ mostrada en (11), se establece la siguiente relación:

$S=U-4 I$

Puesto que la matriz $U$ conmuta con la representación matricial de la DFT - matriz $\underline{\mathbb{F}}-$, es posible afirmar que la matriz $S$ también conmuta con $\underline{\mathbb{F}}$. De esta manera, debe existir un conjunto único y ortogonal de vectores propios tanto de $S$ como de $\mathbb{F}$ (Teorema 2), aunque correspondan a diferentes valores propios (ver comprobación en Dickinson y Steiglitz (1982)). Finalmente, si a partir de (19) se define un operador $\mathcal{S}=$ $\mathcal{D}_{\mathrm{t}}^{2}+\mathcal{F}^{-1} \mathcal{D}_{\omega}^{2} \mathcal{F}$ y se reemplaza $\gamma(t)=\phi_{n}(t)$ (definida en (5)), se puede advertir que las HG-F son las únicas funciones propias de energía finita del operador $\mathcal{S}$ y a su vez son funciones propias del operador $\mathcal{F}$.

Representación matricial de la DFRFT a partir de la matriz de conmutación

Es posible conocer los vectores propios de la matriz $\underline{\mathbb{F}}$ a partir de la estimación de los vectores propios de la matriz de conmutación $\boldsymbol{S}$. Sin embargo, como los vectores propios de la DFT son pares e impares (McClellan y Parks, 1972), no es posible hacer esta estimación directamente sobre $\boldsymbol{S}$ pues sus vectores propios también lo son. Debido a esto, se debe restringir la búsqueda de los vectores propios de $\boldsymbol{S}$ al conjunto de vectores sobre los espacios pares e impares (Candan et al., 2000; McClellan y Parks, 1972). Esto se puede conseguir usando una matriz $B=B^{-1}=B^{T}$ que descompone un vector arbitrario $v[n]$ en sus componentes pares e impares, de tal manera que:

$B_{\text {impar }}=\frac{1}{\sqrt{2}}\left[\begin{array}{ccc}\sqrt{2} & Z_{1, N-1} & \cdots \\ Z_{N-1,1} & I_{l} & J_{l} \\ \vdots & J_{l} & -I_{l}\end{array}\right] ; \quad B_{\text {par }}=\frac{1}{\sqrt{2}}\left[\begin{array}{cccc}\sqrt{2} & Z_{1, N-1} & \cdots & \cdots \\ Z_{N-1,1} & I_{l} & Z_{l, 1} & J_{l} \\ \vdots & Z_{1, l} & \sqrt{2} & Z_{1, l} \\ \vdots & J_{l} & Z_{l, 1} & -I_{l}\end{array}\right]$

donde, $Z_{m, n}$ es una matriz $m \times n$ cuyos elementos son cero, $I_{n}$ es una matriz identidad de orden $n, J_{n}$ es una matriz de ceros excepto su diagonal secundaria cuyos elementos son uno, $l=\{(N / 2)-1\}$ y $\{g\}$ es la función parte entera del escalar $g$. En este caso, $B$ es una matriz simétrica y unitaria que mapea la parte par de un vector de $N$-dimensiones para las primeras $[(N / 2)+1]$ componentes y la parte impar para las componentes restantes. Con ayuda de esta matriz, y sabiendo que $S$ es una matriz real y simétrica que puede diagonalizarse, se puede obtener una matriz $\boldsymbol{A}$ con forma de bloque diagonal:

$A=B S B^{-1}=\left[\begin{array}{cc}E v & 0 \\ 0 & O d d\end{array}\right]$

Donde, $E v$ y $O d d$ son matrices independientes que permiten determinar de forma separada los vectores propios de $\boldsymbol{A}$ y cuyas extensiones par/impar serán los correspondientes vectores propios de $\boldsymbol{S}$. En este punto, los vectores propios de las matrices $E v$ y $O d d$ se deben organizar de acuerdo al orden decreciente de sus respectivos valores propios y se renombran con la notación $\hat{e}_{k}$ y $\hat{o}_{k}$ respectivamente. Finalmente, si se definen las matrices $u_{2 k}$ y $u_{2 k+1}$ que contienen los vectores propios pares e impares de $\boldsymbol{S}$, se tiene que:

$u_{2 k}=B\left[\hat{e}_{k}{ }^{T} ; 0 \ldots 0\right]^{T} ; \quad u_{2 k+1}=B\left[0 \ldots 0 ; \hat{o}_{k}{ }^{T}\right]^{T}$ 
Puesto que las matrices $u_{2 k}$ y $u_{2 k+1}$ definidas en (24) son ortonormales y contienen los vectores propios de matriz $\underline{\mathbb{F}}$, se puede definir la representación matricial del operador DFRFT como:

$\underline{F}^{a}[m, n]=\sum_{k=0, k \neq[(N-1)+N m o d 2]}^{N-1} u_{k}[m]\left(\lambda_{k}\right)^{a} u_{k}[n] ; \quad$ donde $n, m=1,2, \ldots, N$

Finalmente, si $x[k]$ es la representación discreta de una señal de $N$ muestras, la DFRFT por descomposición espectral de dicha señal se define como:

$X_{a}=\underline{F}^{a}[m, n] \cdot x[k]$

Puesto que las HG-F son funciones propias comunes del operador $\mathcal{S}$ (definido en la sección 4) y del operador $\mathcal{F}$, los vectores que conforman la matriz $u_{k}$ pueden ser denominados como la contraparte discreta de las HG-F. Sin embargo, para que esto se cumpla, las HG-F deben ser muestreadas con $T_{S}=\sqrt{2 \pi / N}$ y escaladas por $\sqrt{T_{S}}$ (ver demostración en Pei et al. (1999)). A partir de esta afirmación, la matriz DFRFT definida en (25) puede ser expresada en función de las HG-F muestreadas $\phi_{n}\left(T_{S} k\right)$ de la siguiente manera:

$\underline{\mathbb{F}}^{a}[k, m]=T_{S} \sum_{n=0}^{N-1}\left[\phi_{n}\left(T_{S} k\right)\right]\left(e^{-j n a \pi / 2}\right)\left[\phi_{n}\left(T_{S} m\right)\right]$

\section{CORRESPONDENCIA ENTRE LA FRFT Y LA DFRFT POR DESCOMPOSICIÓN ESPECTRAL}

En esta sección se mostrará la relación que existe entre la FRFT y la DFRFT, de manera que esta última pueda ser usada para obtener resultados similares a la transformada continua dentro de la precisión que provee la aproximación de vectores discretos. Es importante aclarar que el proceso expuesto en esta sección difiere del presentado por Pei et al. (1999), pues se ha evitado emplear lenguaje geométrico para facilitar su comprensión.

Dominio fraccional de Fourier (FRFd) y resolución de la DFRFT

A partir de la definición presentada en (5), las HG-F de orden $n$ con varianza $\sigma$ pueden ser expresadas así:

$h_{n}(t, \sigma)=(1 / \sqrt{\sigma}) \mu_{n} H_{n}(t / \sigma) \exp \left(-t^{2} / 2 \sigma^{2}\right)=(1 / \sqrt{\sigma}) \phi_{n}(t / \sigma)$

Usando la propiedad de escalamiento de la FRFT (ver tabla I en Almeida (1994)), la transformada de orden $a$ de las funciones definidas en (28), es:

$\mathcal{F}^{a}\left[h_{n}(t, \sigma)\right](u)=\sqrt{\sigma}\left\{\sqrt{\frac{1-j \cot \alpha}{1-j \sigma^{2} \cot \alpha}} e^{j \frac{u^{2}}{2} \cot \alpha\left[1-\frac{\cos ^{2} \alpha}{\cos ^{2} \beta}\right]}\right\}\left(e^{-j n b \pi / 2}\right)\left[\phi_{n}\left(\sigma \frac{\sin \beta}{\sin \alpha} u\right)\right]$

donde, $\beta=\tan ^{-1}\left\{(\tan \alpha) / \sigma^{2}\right\}$ y $b=2 \beta / \pi$. Expresando la relación $\sigma(\sin \beta / \sin \alpha)$ con ayuda de la identidad trigonométrica $\left[\sin \beta=\tan \beta / \sqrt{1+\tan ^{2} \beta}\right.$, se tiene:

$\sigma \frac{\sin \beta}{\sin \alpha}=1 /\left(\sqrt{\sigma^{2} \cos ^{2} \alpha+\frac{\sin ^{2} \alpha}{\sigma^{2}}}\right) \equiv \frac{1}{\sigma_{\alpha}}$

La constante $\sigma_{\alpha}$ es definida como la varianza en el FRFd. Al evaluar esta nueva varianza se observa que para $\alpha=0, \sigma_{\alpha}=\sigma$ y para $\alpha=\pi / 2, \sigma_{\alpha}=1 / \sigma$. Si se aplica la definición de (28) y se agrupan los términos encerrados entre corchetes de (29) en la variable $Z(u)$, esta ecuación queda:

$\mathcal{F}^{a}\left[h_{n}(t, \sigma)\right](u)=\sqrt{\sigma} Z(u)\left(e^{-j n b \pi / 2}\right)\left[\sqrt{\sigma_{\alpha}} h_{n}\left(u, \sigma_{\alpha}\right)\right]$

Puesto que las funciones HG-F forman un conjunto ortonormal, una señal discretizada $x(t)=x\left(k T_{S}\right)$ con valor cero fuera del intervalo $\left\{-T_{d}=-(N / 2), T_{d}=(N / 2)-1\right\}$, puede ser extendida en $N$ partes de la siguiente manera: 
$x(t)=\sum_{n=0}^{\infty}\left[T_{S} \sum_{k=-(N / 2)}^{(N / 2)-1} x\left(k T_{S}\right) h_{n}\left(k T_{S}, \sigma\right)\right] h_{n}(t, \sigma) \quad$ donde $N=k=t / T_{S}$

Al calcular la FRFT a ambos lados de (32), la transformada de la señal $x(t)$ es:

$\mathcal{F}^{a}[x(t)](u)=X_{a}(u)=\sum_{n=0}^{\infty}\left[T_{S} \sum_{k=-(N / 2)}^{(N / 2)-1} x\left(k T_{S}\right) h_{n}\left(k T_{S}, \sigma\right)\right] \mathcal{F}^{a}\left[h_{n}(t, \sigma)\right](u)$

Reemplazando el resultado de (31) en (33) y reescribiendo se tiene que:

$X_{a}(u)=\sqrt{\sigma \sigma_{\alpha}} Z(u) \times \sum_{n=0}^{\infty}\left[T_{S} \sum_{k=-(N / 2)}^{(N / 2)-1} x\left(k T_{S}\right) h_{n}\left(k T_{S}, \sigma\right)\right]\left(e^{-j n b \pi / 2}\right)\left[h_{n}\left(u, \sigma_{\alpha}\right)\right]$

Si se define una resolución para la DFRFT $\left(\Delta_{\alpha}=u / m\right)$ en el FRFd y se toman los primeros $N$ términos de la sumatoria de las HG-F, (34) queda:

$X_{a}\left(\Delta_{\alpha} m\right)=\sqrt{\sigma \sigma_{\alpha}} Z\left(\Delta_{\alpha} m\right) \times \sum_{n=0}^{N-1}\left[T_{S} \sum_{k=-(N / 2)}^{(N / 2)-1} x\left(k T_{S}\right) h_{n}\left(k T_{S}, \sigma\right)\right]\left(e^{-j n b \pi / 2}\right)\left[h_{n}\left(\Delta_{\alpha} m, \sigma_{\alpha}\right)\right]$

Multiplicando el lado derecho de (35) por $(\sqrt{N / 2 \pi} \times \sqrt{2 \pi / N})$, usando (28) y reordenando se tiene:

$X_{a}\left(\Delta_{\alpha} m\right)=Z\left(\Delta_{\alpha} m\right)\left(\sqrt{\frac{N}{2 \pi}} T_{S}\right) \sum_{k=-(N / 2)}^{(N / 2)-1} x\left(k T_{S}\right) \times\left\{\sqrt{\frac{2 \pi}{N}} \sum_{n=0}^{N-1}\left[\phi_{n}\left(\frac{k T_{S}}{\sigma}\right)\right]\left(e^{-j n b \pi / 2}\right)\left[\phi_{n}\left(\frac{\Delta_{\alpha} m}{\sigma_{\alpha}}\right)\right]\right\}$

Al comparar la definición de la DFRFT dada en (27) con la sumatoria entre corchetes de (36), estos términos solo pueden ser iguales si $T_{S}=\sqrt{2 \pi / N}$ y además:

$\sigma=T_{S}(\sqrt{N / 2 \pi}) \quad \& \quad \Delta_{\alpha}=\sqrt{T_{S}{ }^{2} \cos ^{2} \alpha+\left(2 \pi / N T_{S}\right)^{2} \sin ^{2} \alpha}$

Si se define la señal discretizada $x\left(k T_{S}\right)=x[k]$ y se reescribe la ecuación (36) con ayuda de (26) se tiene:

$X_{a}\left(\Delta_{\alpha} m\right)=\left[\sigma \cdot Z\left(\Delta_{\alpha} m\right)\right]\left\{\sum_{k=-(N / 2)}^{(N / 2)-1} x[k]\left(\underline{\mathbb{F}}^{b}[k, m]\right)\right\}=\left[\sigma \cdot Z\left(\Delta_{\alpha} m\right)\right]\left\{X_{b}[m]\right\}$

La ecuación (38) expresa que la FRFT continua de orden a puede implementarse mediante una DFRFT de orden $b$ multiplicada por el factor de compensación $\left[\sigma \cdot Z\left(\Delta_{\alpha} m\right)\right]$.

\section{Casos especiales entre FRFT vs DFRFT}

Para obtener una buena aproximación de la FRFT mediante la definición de la DFRFT presentada en (38) se debe tener en cuenta que cuando $T_{S} \approx \sqrt{2 \pi / N}$, la varianza $\sigma \approx 1$ y los efectos de compensación serán muy pequeños, haciendo que $a \approx b$. En este caso, el rango del tiempo $t=N T_{S}$ y el rango de la frecuencia $\omega=2 \pi / T_{S}$ serán prácticamente iguales y la DFRFT aproximará de manera adecuada a la FRFT. Para los casos en los que $T_{S} \neq \sqrt{2 \pi / N}$, se deben seguir los siguientes pasos:

i) Estimar el ángulo de modificación $\beta=\tan ^{-1}\left[\left(2 \pi / N T_{S}^{2}\right)(\tan \alpha)\right]$

ii) Calcular la resolución en el dominio fraccional $\left(\Delta_{\alpha}\right)$ usando (37) y organizar una sucesión de muestras tal que $u_{m}=\Delta_{\alpha} m$. Estas muestras serán los puntos en los que se quiere calcular la FRFT

iii) Calcular la DFRFT de la señal muestreada $x[k]$ para el orden fraccional $b=2 \beta / \pi$, usando la definición presentada en (26)

iv) Multiplicar la DFRFT por el factor de compensación $\left[\sigma \cdot Z\left(\Delta_{\alpha} m\right)\right]$

v) Aplicar la relación de correspondencia dada en (38) 


\section{ALGORITMOS PARA EL CÁLCULO DE LA DFRFT POR DESCOMPOSICIÓN ESPECTRAL}

A partir de los procedimientos presentados en las secciones 4 y 5 , la Figura 1a muestra el algoritmo general para la implementación de la DFRFT por descomposición espectral. Sin embargo, es importante resaltar que en el proceso para calcular la matriz de la DFRFT $\left(\mathbb{F}^{a}\right)$, la matriz de vectores $u_{k}$ siempre será independiente del orden fraccional $a$. Adicionalmente, si el periodo de muestreo $T_{S}$ es muy pequeño, la relación $\sigma=T_{S} \sqrt{N / 2 \pi}$ mostrada en (37), tenderá a cero y hará que el ángulo de modificación $\beta \approx \pi / 2$. Estas observaciones pueden ser empleadas para reducir los errores de cálculo y el costo computacional en la implementación de la DFRFT por descomposición espectral.

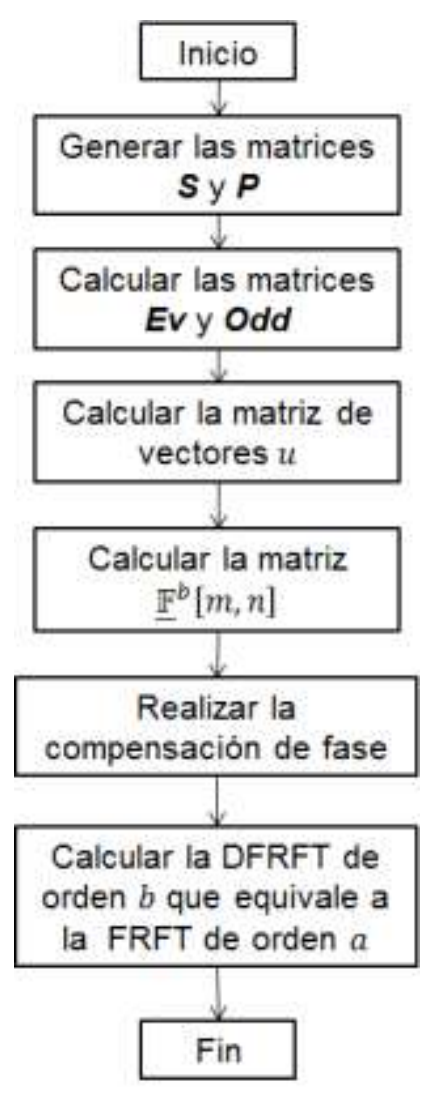

(a)

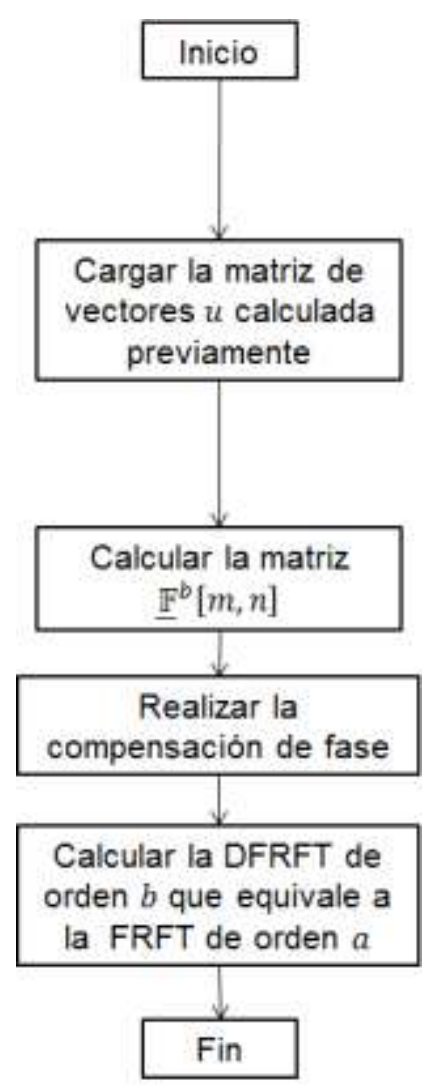

(b)

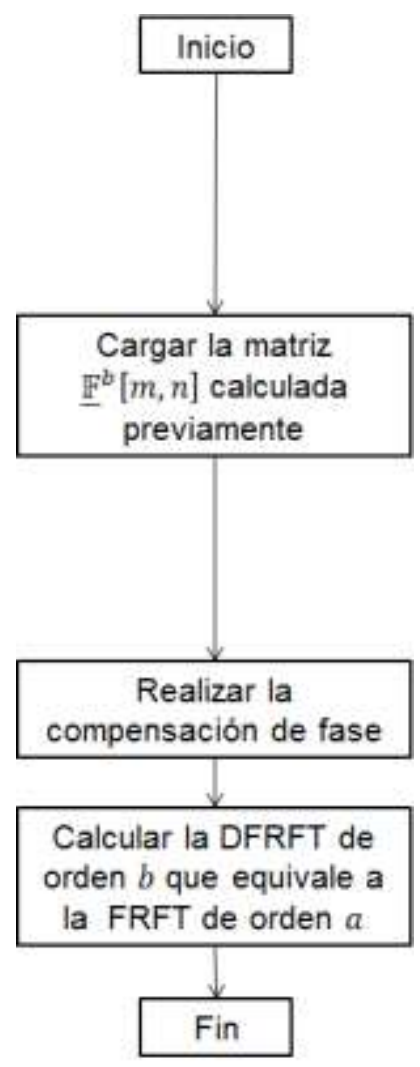

(c)

Fig. 1: Algoritmos de la DFRFT. (a) procedimiento completo; (b) pre-calculando la matriz de vectores propios $u_{k}$; (c) pre-calculando la matriz $\mathbb{F}^{b}[k, m]$

En un gran número de aplicaciones en ingeniería, los datos capturados dependen de la adquisición de la señal de interés dentro una ventana de tiempo pre-establecida con tiempos de muestreo fijos que almacenan una señal discretizada de $N$ cantidad de muestras. Para estos casos, es posible definir un valor fijo de $N$ y así pre-calcular la matriz $u_{k}$ (ver Figura 1b) o la matriz $\mathbb{F}^{b}$ (ver Figura 1c), para luego almacenarlas y usarlas en el cálculo de la DFRFT tantas veces como se desee. Puesto que el cálculo de la matriz $u_{k}$ involucra la búsqueda de los vectores y valores propios de la matriz de conmutación $\mathbf{S}$ y el cálculo de la matriz $\mathbb{F}^{b}$ requiere del producto de matrices $N \times N$, el almacenar estas matrices reduce notablemente la cantidad de operaciones realizadas y por ende los tiempos computacionales, especialmente cuando la cantidad de muestras de la señal bajo estudio es considerable. Además, el almacenamiento de la matriz $u_{k}$ facilita la implementación de la DFRFT cuando esta se usa en aplicaciones en tiempo real, y el almacenamiento de la matriz $\mathbb{F}^{b}$ se justifica cuando el volumen de datos hace la ejecución del algoritmo una tarea muy pesada desde el punto de vista computacional.

La Figura 2 muestra los tiempos computacionales que toma el cálculo de la DFRFT, cuando se aplica el algoritmo completo (algoritmo 1), cuando se guarda previamente la matriz $u_{k}$ (algoritmo 2) y cuando se guarda previamente la matriz $\mathbb{F}^{b}$ (algoritmo 3 ). Para estas simulaciones se empleó un computador de 4 núcleos con velocidad de procesamiento de $2.4 \mathrm{GHz}$ y una memoria RAM de $8 \mathrm{~GB}$. 


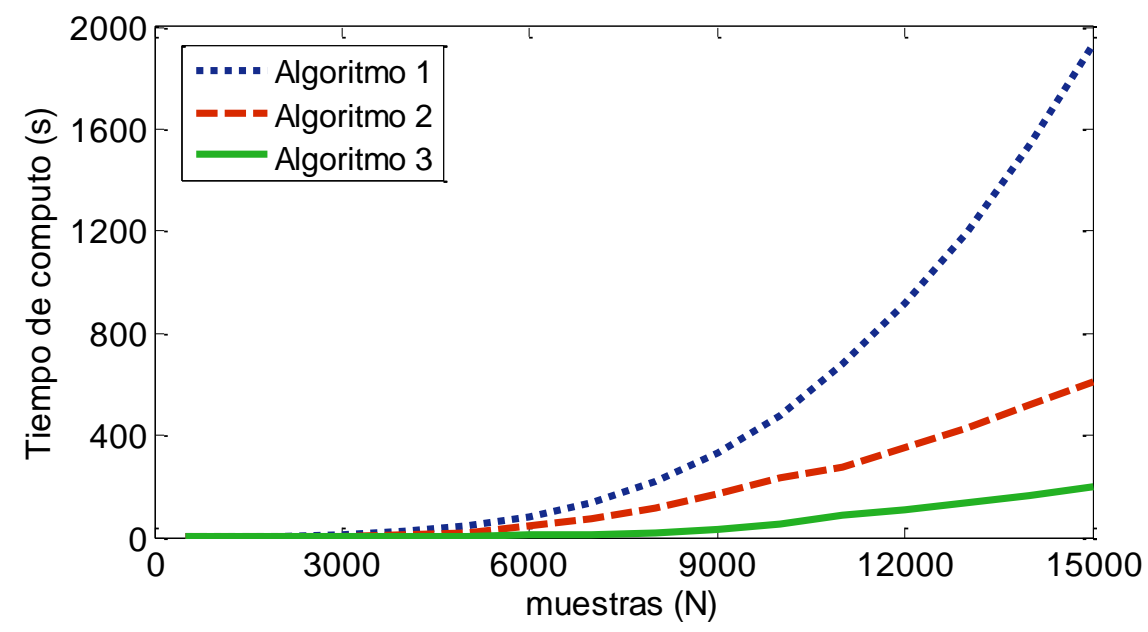

Fig. 2: Tiempos de cómputo para los algoritmos propuestos de la DFRFT

Si se compara el algoritmo completo con los algoritmos modificados propuestos, es evidente cómo la estimación de las matrices $u_{k}$ y $\mathbb{F}^{b}$ consumen la mayor parte del tiempo de cómputo en el algoritmo de la DFRFT. Esta reducción en la cantidad de cálculos realizados justifica el almacenar previamente estas matrices. Estos resultados también permiten hacerse a una idea de los requerimientos de memoria de los algoritmos reducidos comparados con el algoritmo completo.

\section{SIMULACIONES}

Para comprobar que la definición de la DFRFT expuesta en este artículo aproxima de manera adecuada a la FRFT continua (cuarto requerimiento), en esta sección se implementa la DFRFT definida en (38) para algunas señales comunes. En cada caso, se presenta la DFRFT en diferentes órdenes fraccionales $a$ y el caso generalizado cuando $a=1$ el cual corresponde a la FT convencional (dominio de la frecuencia $\omega$ ). Para todas las simulaciones se usan señales discretas de $\mathrm{N}=2000$ muestras.

\section{Señal gaussiana}

Según la literatura, la FRFT de orden $a$ de una señal $x(t)$ también puede ser definida como la rotación de la distribución Wigner (WD) de dicha señal en un ángulo $\alpha=a \pi / 2$ sobre el TFp (Almeida, 1994). Además, se sabe que la WD de una señal gaussiana es una gaussiana de dos dimensiones en el TFp. De esta manera, es de esperarse que la FRFT de cualquier señal gaussiana sea otra señal gaussiana. La Figura 3 muestra la DFRFT de una señal definida matemáticamente como $x(t)=\exp \left(-2 \pi t^{2}\right)$ para varios ordenes fraccionales. Además, se muestra que la FT de una gaussiana es otra gaussiana, lo que confirma la teoría de procesamiento de señales.

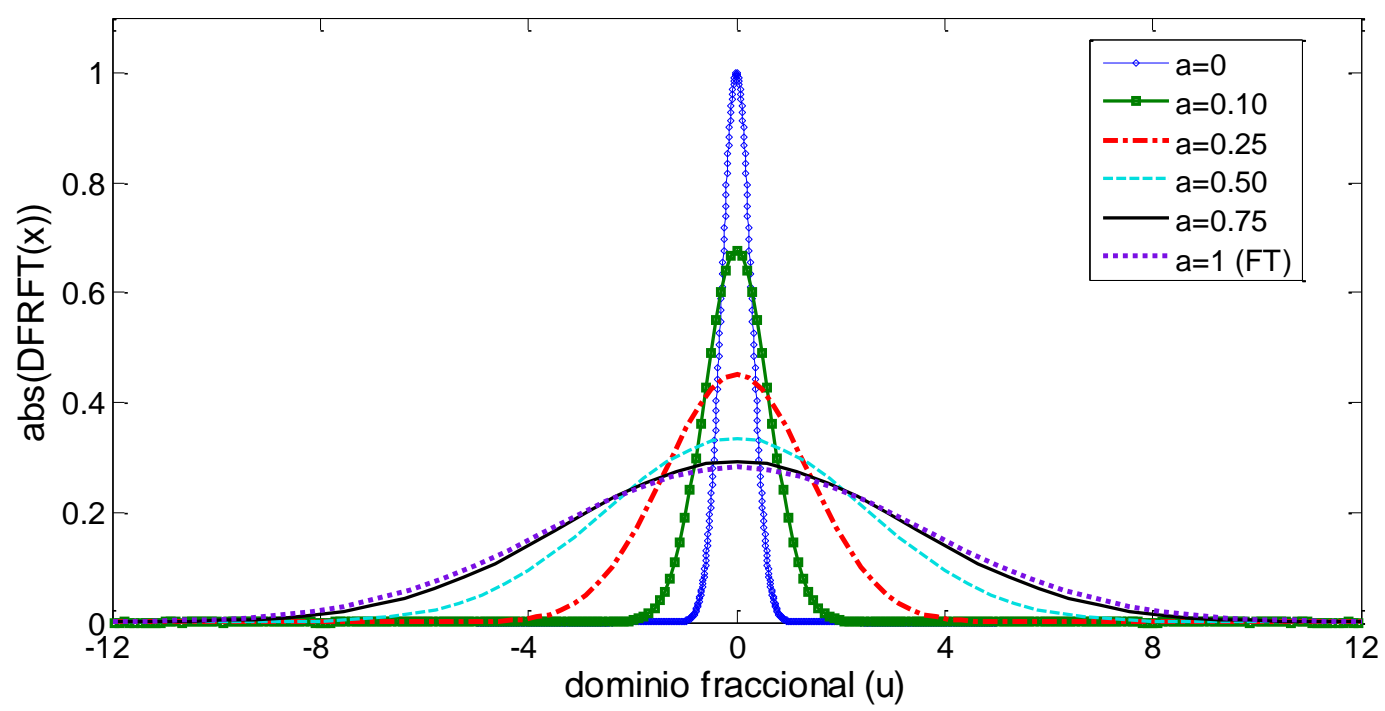

Fig. 3: DFRFT de una señal gaussiana para diferentes órdenes fraccionales 


\section{Señal rectangular}

Es bien sabido que la FT de una señal rectangular es una función seno cardinal $\operatorname{sinc}(f)$. La función rectangular usada es definida como $x(t)=1$ para $t<|1|$ y $x(t)=0$ para el resto. La señal es definida en el intervalo $t \in\{-5,5\}$. La Figura 4 muestra que la DFRFT de un pulso rectangular continuo es una función continua dependiente del orden fraccional $a$. Resulta interesante ver como la DFRFT de una señal rectangular se transforma en una función seno cardinal a medida que el orden fraccional varía entre 0 y 1 . Además, esta prueba confirma que la definición presentada de la DFRFT converge a la FRFT continua, pues cuando $a \approx 0$ el resultado de la DFRFT es la señal original y cuando $a=1$ entrega la FT.
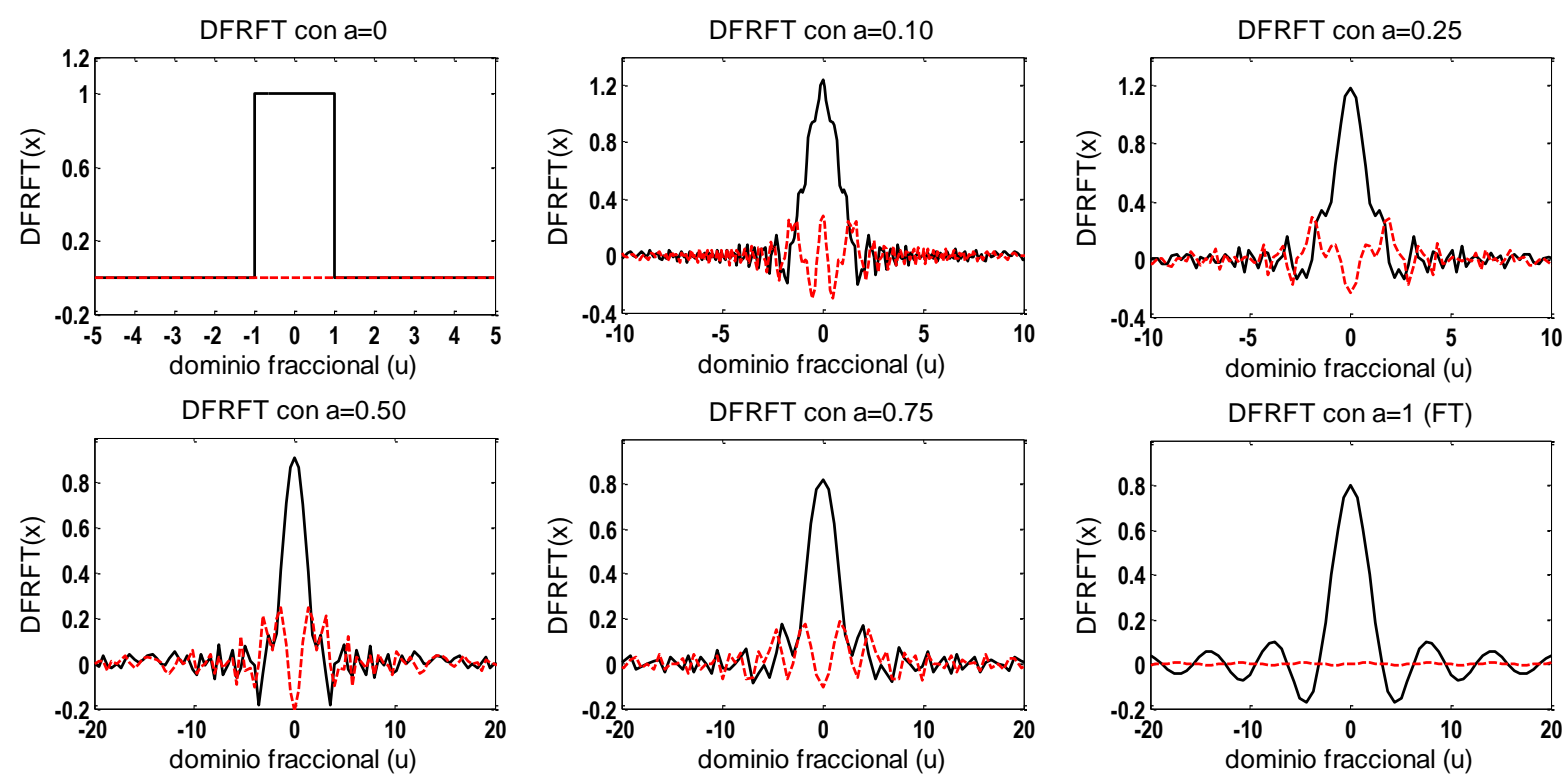

Fig. 4: DFRFT de una señal rectangular para diferentes órdenes fraccionales.

Parte real (línea continua) y parte imaginaria (línea punteada)

\section{Señal senoidal con componentes armónicas}

Según la teoría, la FT de una señal armónica es conjunto de pulsos de Dirac ubicados en las bandas de la frecuencia angular fundamental $\omega_{0}$ y las frecuencias armónicas de la señal. La Figura 5 muestra la DFRFT de una señal con contenido armónico definida como:

$x(t)=\sin \left(\omega_{0} t\right)+0.1 \sin \left(3 \omega_{0} t\right)+0.2 \sin \left(5 \omega_{0} t\right)+0.5 \sin \left(7 \omega_{0} t\right)+0.25 \sin \left(9 \omega_{0} t\right)$

donde, $\omega_{0}=2 \pi f_{0}$ es la frecuencia angular y $f_{0}$ es la frecuencia fundamental, que para este caso es $60 \mathrm{~Hz}$.
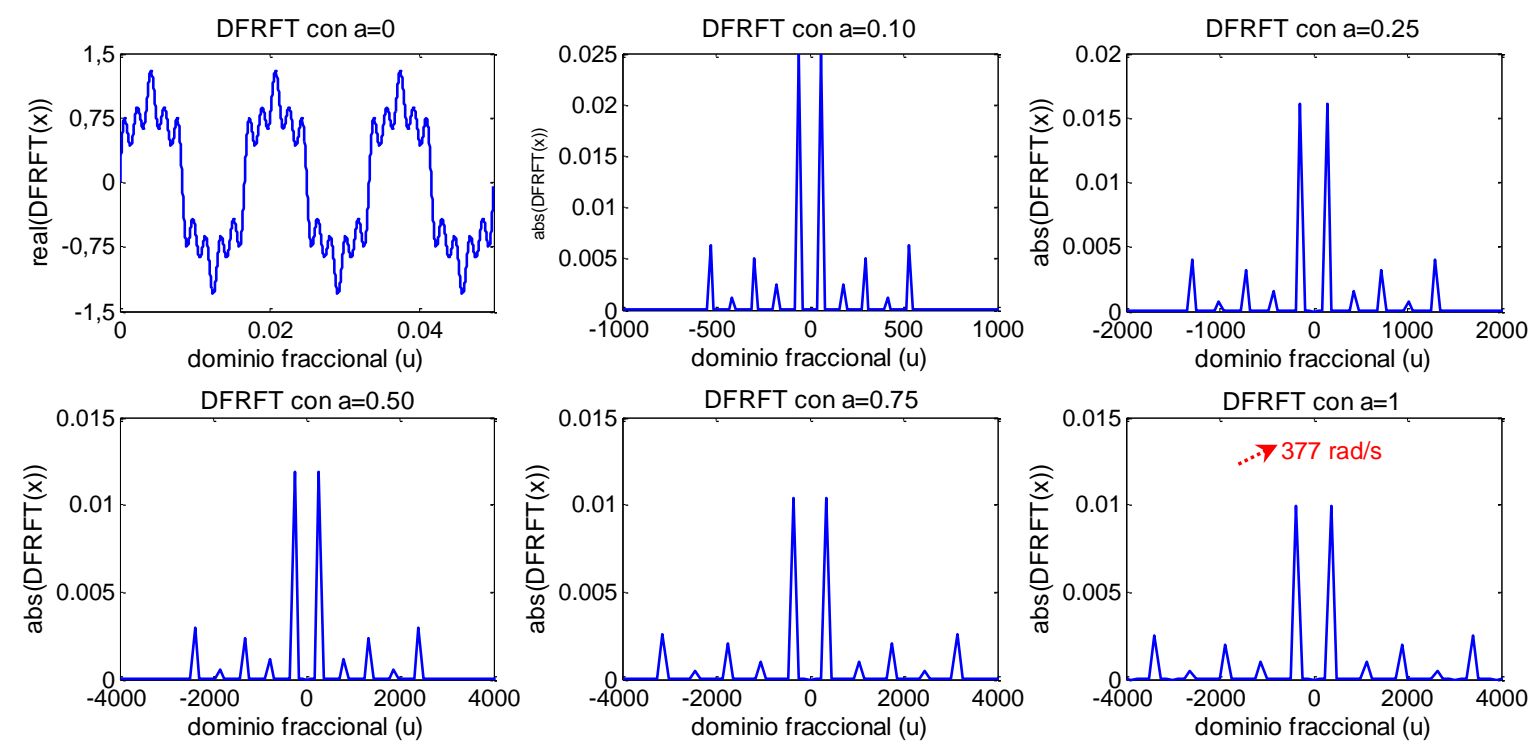
Se puede notar como la DFRFT opera sobre la señal resaltando las componentes armónicas (picos de menor magnitud) para los órdenes fraccionales $a=0.1$ y $a=0.25$, esto es gracias a su propiedad de concentrar la energía de la señal. Ahora, cuando $a=1$ la DFRFT presenta su valor máximo en la banda correspondiente a la frecuencia fundamental de la señal $\left(f_{0}=60 \mathrm{~Hz}\right.$ y $\left.\omega_{0}=377 \mathrm{rad} / \mathrm{s}\right)$ y los picos de menor magnitud en las bandas de frecuencia $3 \omega_{0}, 5 \omega_{0}, 7 \omega_{0}$ y $9 \omega_{0}$. Estos resultados corresponden a la FT de la señal expresada en (39).

\section{CONCLUSIONES}

En este artículo se ha expuesto de manera detallada el proceso para la implementación computacional de la DFRFT por descomposición espectral del operador matricial $\underline{\mathbb{F}}$ de la DFT. Esta definición requiere de la estimación de los valores y vectores propios de $\underline{\mathbb{F}}$ usando una matriz de conmutación (matriz $\boldsymbol{S}$ ).

Se realizaron también algunos aportes y aclaraciones con respecto a los trabajos encontrados en la literatura, de los que se rescatan dos aspectos importantes: primero, los vectores propios de la matriz de conmutación $\boldsymbol{S}$ no son exactamente iguales a las HG-F pues difieren de estos por la constante de escalamiento $\omega_{0}$ mostrada en (20); y segundo, la DFRFT sólo es una aproximación directa de la FRFT para el caso en el que el tiempo de muestreo $T_{S}=\sqrt{2 \pi / N}$. Puesto que no es posible cumplir esta condición para señales muestreadas con un $T_{S}$ pequeño, es necesario incluir un factor de compensación en el cálculo de la DFRFT.

Aunque la DFRFT por descomposición espectral presenta mayores costos y complejidad computacional que otras definiciones de la DFRFT, satisface ampliamente las propiedades de la FRFT continua. Esto permite su uso en mayor variedad de aplicaciones incluyendo filtrado, detección de singularidades y encriptación de información, entre otras. Además, su algoritmo puede ser modificado para reducir los tiempos de cómputo, especialmente cuando se analicen señales con una cantidad considerable de muestras $(N \geq 5000)$. Dichas mejoras se pueden obtener mediante el cálculo y almacenamiento preliminar de la matriz de vectores propios $u_{k}$ (algoritmo 2) o de la matriz $\underline{F}^{b}$ (algoritmo 3). Esta última modificación sólo puede ser implementada en aquellos casos en los que se cumpla la condición $T_{S} \ll \sqrt{2 \pi / N}$.

Finalmente, se han presentado algunos resultados de la implementación computacional de la DFRFT obteniendo una buena aproximación a la FRFT y a su condición generalizada para $a=1$, es decir la FT convencional.

\section{MATERIAL COMPLEMENTARIO}

Este artículo incluye como material complementario el proceso que permite estimar el sistema matricial presentado en la ecuación (20). Este material puede ser solicitado de forma gratuita a los autores (herojasc@udistrital.edu.co).

\section{AGRADECIMIENTOS}

Este trabajo y su publicación han sido financiados por la Dirección de Investigación sede Bogotá (DIB) de la Universidad Nacional de Colombia (proyecto de investigación QUIPU 201010018759) y por la Vicerrectoria de Investigación, Innovación, Creación, Extensión y Proyección Social (VIICEPS) de la Universidad Distrital Francisco José de Caldas a través de su convocatoria 13-2012 (proyecto de investigación 2-5-355-13).

\section{REFERENCIAS}

Alarcón, V., L. Guerrero, J. Rodriguez, y R. Rosas, Análisis de Tráfico Auto-similar en Redes de Comunicaciones Usando Onditas (Wavelets), Información Tecnológica, 16(2), 61-66 (2005).

Almeida, L. B., The fractional Fourier transform and time-frequency representations, IEEE Transactions on Signal Processing, 42(11), 3084-3091 (1994).

Barker, L., The discrete fractional Fourier transform and Harper's equation, Mathematika, 47, 281-297 (2000).

Bracewell, R. N., The Fourier Transform and Its Applications, 3rd Ed., p. 616. New York, USA: McGraw-Hill (2000).

Bultheel, A. y H. Sulbaran, Computation of the fractional Fourier transform, Applied and Computational Harmonic Analysis, 16(3), 182-202 (2004). 
Candan, C., M. A. Kutay, y H. M. Ozaktas, The discrete fractional Fourier transform, IEEE Transactions on Signal Processing, 48(5), 1329-1337 (2000).

Cincotti, G., F. Gori y M. Santarsiero, Generalized self-Fourier functions, Journal of Physics A: Mathematical and General, 25(20), 1191-1194 (1992).

Cruz, C., R. Reyes y H. Perez, Verificación de Firmas Usando Transformada de Gabor, Información Tecnológica, 15(3), 53-60 (2004).

Dickinson, B. y K. Steiglitz, Eigenvectors and functions of the discrete Fourier transform, IEEE Transactions on Acoustics, Speech, and Signal Processing, 30(1), 25-31 (1982).

Elaydi, S., An Introduction to Difference Equations, 3rd Ed., p. 568. New York, USA: Springer (2005).

Hsu, H., Theory and Problems of Signals and Systems, p. 466. New York, USA: McGraw-Hill (1995).

Koc, A., H. Ozaktas y M. Kutay, Digital Computation of Linear Canonical Transforms, IEEE Transaction on Signal Processing, 56(6), 2383 - 2394 (2008).

Lebedev, N., Special functions and their applications, Revised Ed., p. 336. New York, USA: Dover Publications (1972).

Mantilla, I., Análisis numérico, 1ra Ed., p. 302. Bogotá, Colombia: Unibiblos (2004).

Martinez, A., Z. Pineda y A. Ramos, Ocultamiento del Mensaje de Señales de Voz usando la Transformada de Ondita Haar Discreta, Información Tecnológica, 21(3), 135-140 (2010).

McBride, A. C. y H. F. Kerr, On Namias's Fractional Fourier Transforms, IMA Journal of Applied Mathematics, 39(2), 159-175 (1987).

McClellan, J. y T. Parks, Eigenvalue and eigenvector decomposition of the discrete Fourier transform. IEEE Transactions on Audio and Electroacoustics, 20(1), 66-74 (1972)

Namias, V., The Fractional Order Fourier Transform and its Application to Quantum Mechanics, IMA Journal of Applied Mathematics, 25(3), 241-265 (1980).

Ozaktas, H., B. Barshan, D. Mendlovic y L. Onural, Convolution, filtering, and multiplexing in fractional Fourier domains and their relation to chirp and wavelet transforms, Journal of the Optical Society of America A, 11(2), 547 (1994).

Ozaktas, H., O. Arikan, M. A. Kutay y G. Bozdagi, Digital computation of the fractional Fourier transform, IEEE Transactions On Signal Processing, 44(9), 2141-2150 (1996).

Pei, S., M. Yeh y C. Tseng, Discrete fractional Fourier transform based on orthogonal projections, IEEE Transactions on Signal Processing, 47(5), 1335-1348 (1999).

Pei, S. C. y J. J. Ding, Closed-form discrete fractional and affine Fourier transforms, IEEE Transactions on Signal Processing, 48(5), 1338-1353 (2000).

Pei, S., W. Hsue y J. Ding, Discrete Fractional Fourier Transform Based on New Nearly Tridiagonal Commuting Matrices, IEEE Transaction on Signal Processing, 54(19), 3815 - 3828 (2006).

Pei, S., W. Hsue, Random Discrete Fractional Fourier Transform, IEEE Signal Processing Letters, 16(12), 1015 - 1018 (2009).

Roman, S., Advanced Linear Algebra, 3rd Ed., p. 526. New York, USA: Springer (2010).

Santamaria, F., C. Cortés y F. Román, Uso de la Transformada de Ondeletas (Wavelet Transform) en la Reducción de Ruidos en las Señales de Campo Eléctrico producidas por Rayos, Información Tecnológica, 23(1), 65-78 (2012).

Sejdić, E., I. Djurović y L. Stanković, Fractional Fourier transform as a signal processing tool: An overview of recent developments, Signal Processing, 91(6), 1351-1369 (2011).

Tao, R., B. Deng y Y. Wang, Research progress of the fractional Fourier transform in signal processing, Science in China: Series F Information Sciences, 49(1), 1-25 (2006).

Tao, R., F. Zhang y Y. Wang, Research progress on discretization of fractional Fourier transform, Science in China, Series F: Information Sciences, 51(7), 859-880 (2008). 